\title{
Protée
}

\section{Éloge de la noirceur}

\section{Claude Zilberberg}

Volume 31, numéro 3, hiver 2003

\section{Lumières}

URI : https://id.erudit.org/iderudit/008436ar

DOI : https://doi.org/10.7202/008436ar

Aller au sommaire du numéro

\section{Éditeur(s)}

Département des arts et lettres - Université du Québec à Chicoutimi

\section{ISSN}

0300-3523 (imprimé)

1708-2307 (numérique)

Découvrir la revue

\section{Citer cet article}

Zilberberg, C. (2003). Éloge de la noirceur. Protée, 31(3), 43-55. https://doi.org/10.7202/008436ar

\section{Résumé de l'article}

L’étude présentée s’efforce de composer raisonnablement un point de vue en ajustant, les unes aux autres, plusieurs exigences pressantes : (i) l'injection de l'intensité dans les structures élémentaires de la signification, injection qui invite à concevoir les traits sémantiques d'abord comme des vecteurs ; (ii) la prise de distance moins à l'égard de la narrativité stricto sensu qu'à l'égard de la narrativisation de la signification, ce qui est bien différent ; (iii) l'injection de l'intensité permet d'" armer " la notion de paradigme et de la doter du ressort qui lui manque encore ; (iv) le rapprochement entre la rhétorique et la sémiotique, lequel jusqu'à un certain point " va de soi ", puisque la rhétorique vise, si l'on en croit les bons auteurs, à " donner de la vivacité, de la force ou de la grâce au discours "; (v) enfin, pour ce qui est de l'adéquation, l'objet ici considéré, la noirceur, relève du non verbal, mais le plan du contenu s'avérant - jusqu'à preuve du contraire - commun, la différence entre les sémiotiques verbales et les sémiotiques non verbales n'est plus qu'une affaire de pondération, de balance entre les catégories contrôlant l'espace tensif.
Ce document est protégé par la loi sur le droit d'auteur. L'utilisation des services d'Érudit (y compris la reproduction) est assujettie à sa politique d'utilisation que vous pouvez consulter en ligne.

https://apropos.erudit.org/fr/usagers/politique-dutilisation/ 


\title{
ÉLO GE DE LA N O IRCEU R
}

\author{
Claude Zilberberg
}

[...] le noir est une énergie.

Paul Klee

\begin{abstract}
À propos du parcours suivi par la sémiotique, on peut, sans grand risque d'erreur, affirmer deux choses: (i) elle a accordé à la narrativité proppienne une importance déterminante au point de lui confier la direction du sens, de sorte que l'on doit, selon le cas, blâmer ou féliciter Greimas d'avoir procédé à une narrativisation de la signification; du même coup, la sémiotique se montrait au mieux indifférente à la rhétorique, laquelle avait pourtant, aux yeux des Anciens et des Modernes en Europe, dirigé et contrôlé le discours pendant plus de deux millénaires. De sorte que l'on peut se demander si la «dénarrativisation» en cours de la signification n'appelle pas - sur nouveaux frais - un rapprochement entre la rhétorique et la sémiotique. En effet, le point de vue tensif, qui accorde à l'intensité la prééminence, ne peut que souscrire aux vues de du Marsais: «[...] les figures, quand elles sont employées à propos, donnent de la vivacité, de la force ou de la grâce au discours; $[\ldots]{ }^{1}$, ou à celles de Fontanier:

Mais les Tropes ont lieu, ou par nécessité et par extension, pour suppléer aux mots qui manquent à la langue pour certaines idées, ou par choix et par figure, pour présenter les idées sous des images plus vives et plus frappantes que leurs signes propres. ${ }^{2}$
\end{abstract}

Sans doute ces notions de "force», de "grâce», de "vivacité» sont-elles loin d'être immédiatement claires, mais il s'en faut que cette objection soit péremptoire; (ii) la sémiotique considère que les catégories qu'elle propose sont pertinentes pour les discours verbaux comme pour les discours non verbaux. C'est à ce double titre: primat de l'intensité et «bonne volonté» du plan de l'expression, que nous avons été amené à nous intéresser à certains aspects du traitement sémiotique de la lumière.

\section{LES STRUCTURES CANONIQUES}

La prise de distance à l'égard de la narrativité - moins d'ailleurs à l'égard de la narrativité qu'à l'égard de l'extension qui lui a été concédée - et le rapprochement 
avec la rhétorique supposent la prise en compte de catégories distinctes de celles qui ont assuré jusqu'à maintenant la narrativisation de la signification.

\subsection{Les structures étendues}

L'identification d'un pivot discursif solide ne va pas de soi: dans la Rhétorique, Aristote propose une catégorisation des discours, selon les situations où ils sont tenus, et un pivot: l'enthymème, c'est-à-dire un syllogisme tronqué. Si nous recevons cette grandeur comme implicative, et compte tenu du fait qu'elle ne fait pas sens à elle seule, reste à l'apparier. Si, sans chercher malice, nous recevons l'implication comme une relation nécessaire et de droit, son corrélat doit se présenter comme non nécessaire et, de fait, parmi les outils que les grammaires mettent à notre disposition, la concession est certainement la fonction qui satisfait le moins mal à cette double demande. À l'appui de cette hypothèse, cette tension: implication versus concession, n'est pas sans rappeler l'inégalité que Hjelmslev relève entre, d'une part, la "détermination" et l'«interdépendance», d'autre part, la "constellation" ${ }^{3}$. Nous attendons de cette tension qu'elle éclaire l'affinité entre le discours et l'événement, puisque la «vivacité» de ce dernier décide, aux yeux de l'énonciataire, de l'intérêt ou de la platitude du discours qui lui est tenu, c'est-à-dire, en fin de compte, de la passation ou de la non-passation du contrat discursif. Mais ce cadre très général risque de demeurer vide, s'il n'est pas relayé par des structures locales.

\subsection{Les structures locales}

Malgré la référence à Hjelmslev, l'épistémologie de la sémiotique croit devoir toujours distinguer entre le contenu des grandeurs et les opérations affectant ces grandeurs, bref entre morphologie et syntaxe. Selon une présentation suggestive empruntée à Bachelard, il s'agit de renoncer au participe passé, au trait, en faveur du participe présent, du vecteur ${ }^{4}$. Ou plutôt, la fonction sémiotique s'imposant aussi au métalangage, la fixité des grandeurs concerne le plan de l'expression, leur fluence, le plan du contenu.

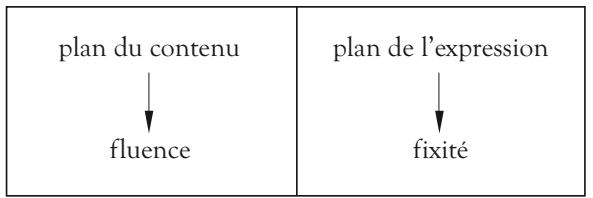

Les grandeurs sémiotiques chiffreraient des fluences directionnelles et aspectuelles, c'est-à-dire mesurables: (i) au titre de la direction, selon l'ascendance ou la décadence; sous une précision d'importance toutefois: l'ascendance et la décadence d'une fluence étant dans la dépendance d'un point de vue, ou d'un accent, un ralentissement par exemple vaut, selon le cas, comme diminution de la célérité, ou bien: comme accroissement de la lenteur; (ii) au titre de l'aspect, nous adaptons la distinction reçue [imperfectif versus perfectif] de manière à faire apparaître en clair l'accroissement ou le décroissement de la fluence; sous cette précision, nous convenons d'analyser - en l'acception hjelmslevienne du terme - l'ascendance en relèvement puis redoublement, le redoublement présupposant le relèvement; pour la décadence, nous mettons en place le couple atténuation puis amenuisement.

Si un couple suffit dans la perspective binariste, en sémiotique les «bonnes" structures demandent un couple de couples permettant aux grandeurs de s'opposer en tant que membres d'un couple, $\left[a_{1} \& a_{2}\right]$ et $\left[b_{1} \& b_{2}\right]$, et de s'opposer entre elles, soit $\left[a_{1}\right.$ vs $\left.a_{2}\right]$ et $\left[b_{1}\right.$ vs $\left.b_{2}\right]$; ce faisant, la véritable complexité se trouve posée, non pas celle qui constate l'existence d'une pluralité, mais celle qui résulte, ainsi que le recommande Hjelmslev dans le neuvième chapitre des Prolégomènes, d'une "intersection de faisceaux de rapports", soit le dispositif hiérarchique suivant:

\begin{tabular}{|c|c|c|}
\hline fonction & \multicolumn{2}{|c|}{ phorie } \\
\hline $\begin{array}{l}\text { dérivés de } \\
1^{\text {er }} \text { degré }\end{array}$ & \multicolumn{2}{|c|}{ décadence versus ascendance } \\
\hline $\begin{array}{l}\text { dérivés de } \\
2^{\mathrm{e}} \text { degré }\end{array}$ & $\begin{array}{l}\text { atténuation versus } \\
\text { amenuisement }\end{array}$ & $\begin{array}{l}\text { relèvement versus } \\
\text { redoublement }\end{array}$ \\
\hline
\end{tabular}

Nous l'avons dit: nous concevons les grandeurs moins comme des traits que comme des phases, et 
comme elles sont divisibles, la récursivité peut opérer et opposer l'engagement de l'atténuation: $\left[s_{1}\right]$ et son aboutissement: $\left[s_{2}\right]$; de même pour l'amenuisement: $\left[s_{3}\right]$ puis $\left[s_{4}\right]$. Les mêmes données valent, mais en sens inverse, pour l'ascendance:

\begin{tabular}{|l|c|c|c|}
\hline $\mathrm{s}_{1}$ & $\mathrm{~s}_{2}$ & $\mathrm{~s}_{3}$ & $\mathrm{~s}_{4}$ \\
\hline atténuation $\longrightarrow$ amenuisement \\
\hline redoublement $\longrightarrow$ relèvement \\
\hline
\end{tabular}

$\left[s_{1}\right],\left[s_{2}\right],\left[s_{3}\right]$ et $\left[s_{4}\right]$ interviennent comme dérivés de troisième degré.

Ces dérivés constituent une structure. Pour désigner les grandeurs ainsi générées, nous retiendrons le terme de contraire, puis, afin de dissimiler la contrariété, nous ferons appel à un terme proposé par Sapir: celui de sous-contraire pour $\left[\mathrm{s}_{2}\right]$ et $\left[s_{3}\right]$, que nous couplons avec celui de sur-contraire pour $\left[s_{1}\right]$ et $\left[s_{4}\right]$. Les sur-contraires s'opposent aux souscontraires et chaque constituant s'oppose encore à son vis-à-vis. Ce faisant, chacun des dérivés de troisième degré reçoit une quadruple définition: tensive au titre de la phorie, directionnelle par présupposition du premier degré, aspectuelle par présupposition du deuxième degré, positionnelle au titre du troisième degré. Soit maintenant:

\begin{tabular}{|c|c|c|c|}
\hline $\mathrm{s}_{1}$ & $\mathrm{~s}_{2}$ & $\mathrm{~s}_{3}$ & $\mathrm{~s}_{4}$ \\
\hline sur-contraire & sous-contraire & sous-contraire & sur-contraire \\
\hline
\end{tabular}

Dans le huitième chapitre des Prolégomènes, Hjelmslev recommande de "procéder par définitions à outrance», ce qui se comprend du reste: les définissantes ne sont pas autre chose que les catégorisantes déclarées par la théorie, mais localisées, greffées sur un signifiant verbal ou non verbal commode. Nous nous proposons de mettre à l'épreuve les hypothèses avancées à propos du traitement de la lumière, c'est-à-dire de la tonicité lumineuse.

\section{LA TONICITÉ ÉMETTRICE}

Selon la convention adoptée plus haut, la tonicité lumineuse renvoie au traitement de la lumière dans l'œuvre, c'est-à-dire au ton dans la terminologie de J. Itten: «Si nous voulons déterminer le degré de clarté ou d'obscurité d'une couleur, nous parlons de la valeur de sa tonalité " 5 .

\subsection{La structure élémentaire de la tonicité émettrice}

Pour mettre en place la structure élémentaire de la luminosité picturale, nous partirons du terme /obscur/ que le Micro-Robert aborde ainsi: "Qui est privé (momentanément ou habituellement) de lumière». Nous recevons /obscur/ comme un surcontraire atone et par mise en ouvre du relèvement, puisque les termes ne tiennent qu'en vertu des opérations qu'ils subsument. Nous recevons /sombre/ , défini lui comme: "Qui est peu éclairé, reçoit peu de lumière", au titre de sous-contraire atone. Par engagement du redoublement, c'est-à-dire ajout d'un quantum de lumière, /clair/, défini comme "Qui a l'éclat du jour, reçoit beaucoup de lumière», s'inscrit, au voisinage de /sombre/, comme sous-contraire tonique. Le sur-contraire de tonicité, étant tenu de changer la superlativité en excessivité, est /éblouissant/ - le Micro-Robert définit "éblouir" comme «Troubler (la vue ou une personne dans sa vision) par un éclat insoutenable» - qui, en français, occupe la position de sur-contraire tonique. En raison de leur dépendance à l'égard des intervalles qui les obligent et si aucun syncrétisme n'intervient, les surcontraires et les sous-contraires sont, sous l'autorité de l'intervalle dont ils ne sauraient se séparer, symétriques et inverses les uns des autres.

\begin{tabular}{|c|c|c|c|}
\hline éblouissant & clair & sombre & obscur \\
\hline $\begin{array}{c}\text { sur-contraire } \\
\text { tonique }\end{array}$ & $\begin{array}{c}\text { sous-contraire } \\
\text { tonique }\end{array}$ & $\begin{array}{c}\mathrm{s}_{3} \\
\text { sous-contraire } \\
\text { atome }\end{array}$ & $\begin{array}{c}\mathrm{s}_{4} \\
\text { sur-contraire } \\
\text { atone }\end{array}$ \\
\hline \multicolumn{2}{|c|}{$\longrightarrow$ atténuation } \\
redoublement
\end{tabular}


Cette mise en place est à la fois naive et problématique. Elle est naïve en ce sens que, si l'on propose à un écolier même fort jeune ce groupe d'«adjectifs» en désordre, il n'aura aucune peine, si on le lui demande, à les ordonner selon l'ascendance ou la décadence, mais cette objection, si c'en est une, pourrait être adressée également à la narrativité proppienne que les collégiens, comme on sait, n'ont aucune peine à manier. Elle est problématique en ce sens que cette déclinaison, quelle que soit son orientation, repose sur plusieurs présupposés: (i) le jeu combiné de la segmentation et de la circulation de la tonicité lumineuse; (ii) la quantification de la qualité, puisque le degré de clarté mesure la quantité de lumière reçue; (iii) la dévolution de l'aspectualité grammaticale aux opérations de relèvement et de redoublement, dans le cas de l'ascendance, de l'atténuation et de l'amenuisement, dans le cas de la décadence, la seconde opération de chaque couple prenant le relais de la première, lorsque celle-ci a ellemême atteint son terme, sinon son chiffre propre:

\begin{tabular}{|l|c|}
\hline décadence & $\begin{array}{c}\text { atténuation } \approx \text { de } s_{1} \text { à } s_{2} \\
\text { puis } \\
\text { amenuisement } \approx \text { de } s_{3} \text { à } s_{4}\end{array}$ \\
\hline ascendance & $\begin{array}{c}\text { relèvement } \approx \text { de } s_{4} \text { à } s_{3} \\
\text { puis } \\
\text { redoublement } \approx \text { de } s_{2} \text { à } s_{1}\end{array}$ \\
\hline
\end{tabular}

(iv) enfin, la résolution figurale de l'excès et du manque par rabattement des deux intervalles canoniques l'un sur l'autre: projection du manque en discours lorsque l'intervalle $\Delta_{2}$, qui mesure la distance entre les sous-contraires, est projeté sur $\Delta_{1}$ et, corrélativement, projection de l'excès en discours lorsque l'intervalle $\Delta_{1}$, qui mesure la distance entre les sur-contraires, est projeté sur $\Delta_{2}$. En ce sens, le MicroRobert n'a pas tort, à nos yeux du moins, de préciser que l'éclat de la lumière dans le cas de l'/éblouissant/ est « insoutenable».

Du point de vue syntaxique, l'ascendance et la décadence deviennent tour à tour objet l'une pour l'autre: (i) si la visée est celle de la décadence, alors le sujet opérateur atténue un redoublement ou amenuise un relèvement, selon le cas; (ii) si la visée est celle de l'ascendance, alors le sujet opérateur relève un amenuisement ou redouble une atténuation, en délivrant - soudainement ou "en prenant son temps» - un surcroît de plus. Ainsi dans Éloge de l'ombre, Tanizaki dénonce comme excessive l'attirance des Occidentaux pour l'/étincelant/:

D'une manière générale, la vue d'un objet étincelant nous procure un certain malaise. Les Occidentaux usent, même pour la table, d'ustensiles d'argent, d'acier, de nickel, qu'ils polissent afin de les faire briller, alors que, nous autres, nous avons en horreur tout ce qui resplendit de la sorte. 6

Plusieurs passages du texte de Tanizaki permettent d'identifier $\left[\mathrm{s}_{2}\right]$ comme l'attracteur prévalent dans son propre discours:

Non point que nous ayons une prévention a priori contre tout ce qui brille, mais, à un éclat superficiel et glacé, nous avons toujours préféré les reflets profonds, un peu voilés; soit, dans les pierres naturelles aussi bien que dans les matières artificielles, ce brillant légèrement altéré qui évoque irrésistiblement les effets du temps. ${ }^{7}$

Dans les termes de Wölfflin, le dilemme est celui-là même qui n'a cessé de l'obséder: "clarté absolue» ou "clarté relative»?

Le paradigme que nous venons de proposer n'est pas tout à fait celui des peintres, sous deux considérations au moins: (i) à notre couple de souscontraires [clair versus sombre], les peintres et l'usage préfèrent le couple [clair versus foncé]; ceci n'est pas trop dommageable, puisque le Micro-Robert définit "foncé» comme ce "qui est d'une nuance sombre (couleur)»; (ii) la question des vernis et des glacis hier, celle des acryliques aujourd'hui, pour être traitée, demande que l'on distingue entre deux tonicités en contraste syntaxique l'une avec l'autre: une tonicité émettrice et une tonicité réflectrice, la première sous le signe de la donation, la seconde sous le signe de la restitution; le couple reçu [clair versus foncé] appartient à la tonicité émettrice.

Nous y reviendrons. 
Ce couple [clair versus foncé] fournissant la souscontrariété, il nous incombe de préciser les termes de la sur-contrariété. Le point délicat, quand il est question de décliner un paradigme, est la reconnaissance de l'ascendance; en effet, notre propre postulat est le suivant: la juste direction est celle qui vise ou saisit le sens comme croissant du point de vue de la (ou des) valence(s) agitée(s). Pour le cas qui nous occupe, la question se laisse aisément formuler: l'intervalle de sous-contrariété [clair versus foncé] étant acquis, des deux directions possibles, [clair $\rightarrow$ foncé] et [foncé $\rightarrow$ clair], quelle est celle qui doit être reconnue comme ascendante? Nous demanderons la réponse à une autorité en la matière, Goethe: «Pour naître, la couleur exige lumière et obscurité, clarté et ombre ou, si l'on veut user d'une expression plus générale, lumière et non-lumière ${ }^{8}$. Si cette affirmation demeure indécidable, la suite met fin à notre dilemme: «Mais chacune des deux [couleurs: le jaune et le bleu] peut, si elle est concentrée ou assombrie, produire elle-même un nouveau phénomène " 9 . La direction croissante nous est révélée par la concentration et, par voie de conséquence, l'assombrissement se saisit de l'ascendance et dénonce, du point de vue tensif, l'éclairement comme déperdition et décadence.

\section{2 Élection de l'assombrissement}

Cette positivité, sinon cette poétique de l'assombrissement, est l'un des motifs récurrents du maître-livre de Tanizaki, notamment dans les passages qu'il consacre à ce qu'il faudrait appeler la culture de la laque:

[...] de tout temps la surface des laques avait été noire, brune ou rouge, autant de couleurs qui constituaient une stratification de je ne sais combien de "couches d'obscurité", qui faisaient penser à quelque matérialisation des ténèbres environnantes. 10

L'obscur se révélant pour Gothe le siège d'un processus de "concentration", pour Tanizaki d'un processus de «stratification», nous faisons nôtre cette orientation et nous recevons / profond/ comme le superlatif de /foncé/; c'est dans cet esprit qu'autrefois le pourpre était reçu comme un rouge profond ou, ce qui revient au même, tonique. Nonobstant l'usage, le terme exsangue de la série décroissante est, si l'on ose dire, le /pâle/, de sorte que /profond/ et/pâle/ s'inscrivent comme sur-contraires de tonicité; les bonnes structures étant asymétriques, /profond/ prévaut, c'est-à-dire qu'il oblige les autres termes à se déterminer par rapport à lui, soit maintenant:

\begin{tabular}{|c|c|c|c|}
\hline $\begin{array}{c}\mathrm{s}_{1} \\
\downarrow\end{array}$ & $\mathrm{s}_{2}$ & $\mathrm{~s}_{3}$ & $\mathrm{~s}_{4}$ \\
profond & foncé & clair & pâle \\
\hline \multicolumn{2}{|c}{ tonicité croissante $\longrightarrow$ atonie croissante } \\
\hline
\end{tabular}

Les structures, ici plastiques, que nous mettons en place, sont justiciables de deux approches distinctes: (i) une approche transitionnelle, sériale, placée sous le signe de la progression ou de la dégression, approche qui admet les glissements affectant soit de degré à limite, soit de degré en degré; nous la désignerons comme modèle de la série; (ii) une approche commutative, alternative, qui n'est pas sans rappeler celle de l'inamovible quatrain à rimes embrassées propre à la poésie française, c'est-à-dire qu'elle impose la coprésence de deux rimes sémantiques, c'est-à-dire de deux espaces pour ainsi dire cloisonnés et tels qu'une entrée dans un espace présuppose une sortie de l'espace mitoyen; dans ce cas, /clair/ «rime» avec /foncé/, /profond/ "rime» avec/pâle/; nous retenons pour ce second cas la dénomination de réseau. Selon Goethe, la seconde approche, qui semble la gardienne de la stabilité des intervalles, convient à l'esthésie visuelle:

L'œil ne peut ni ne veut se maintenir un seul instant dans l'état

uniforme spécifiquement déterminé par l'objet. Une tendance à une sorte d'antagonisme le contraint qui, opposant l'extrême à

l'extrême, l'intermédiaire à l'intermédiaire, réunit instantanément les contraires et s'efforce de constituer une totalité aussi bien quand les phénomènes se succèdent que lorsqu'ils coexistent dans le temps ou dans l'espace. 11

La série et le réseau se présentent comme deux styles syntaxiques possibles pour les grandeurs admises 
ou accédant dans le champ de présence: (i) la série ferait prévaloir la synérèse sur la diérèse, le mélange sur le tri, les valeurs d'univers sur les valeurs d'absolu; (ii) le réseau préférerait la diérèse à la synérèse, le tri au mélange, les valeurs d'absolu aux valeurs d'univers:

\begin{tabular}{|c|c|}
\hline \multicolumn{1}{|c|}{ série } & réseau \\
\hline $\begin{array}{c}\text { synérèse } \\
\text { [mélange] }\end{array}$ & $\begin{array}{c}\text { diérèse } \\
\text { [tri] }\end{array}$ \\
\hline $\begin{array}{c}\text { valeurs } \\
\text { d'univers }\end{array}$ & $\begin{array}{c}\text { valeurs } \\
\text { d'absolu }\end{array}$ \\
\hline
\end{tabular}

\subsection{Productivité de la noirceur}

La confrontation de la blancheur et de la noirceur, de la lumière et de la non-lumière s'avère ainsi singulière. Rappelons d'abord que la singularité n'est pas un écart par rapport au système, une échappée hors du système, mais bien une propriété du système. Inscrite dans le système, la singularité est munie de deux traits: du point de vue figuratif, elle survient et définit pour ce discours ce qui vaut comme événement; du point de vue figural, elle réalise la concession, laquelle attend comme embusquée son intervenir, son survenir. Pour ce qui regarde la tonicité lumineuse, nous nous sommes vite rendu compte, à la lecture des commentaires les plus sagaces, que, si pour la doxa la tonicité est du côté de la /clarté/, pour les "grands astreignants» la tonicité est du côté de l'/obscur/. Selon ces derniers, l'/obscur/, le /foncé/, ne saurait menacer ou nuire au /clair/ puisqu'il le produit!

L'appartenance des sub-valences à un réseau et la coexistence des sur-contraires qu'elle intime sont un des chapitres majeurs de l'imaginaire humain. Selon le style implicatif, les sur-contraires sont censés s'exclure; ils sont dans l'obédience de l'inflexibilité du $o u$, de sorte que la blancheur et la noirceur ne sauraient être dites ensemble d'une même grandeur. Ou plus strictement sans doute: la catalyse de la blancheur, en tant que grandeur distincte, à partir de la noirceur, de même que la catalyse inverse, n'est pas licite pour ce discours. Pourtant, dans La Terre et les rêveries $d u$ repos, G. Bachelard fait l'éloge d'une «[...] image qui vit de la contradiction d'une substance et de son attribut. Dans un sonnet, Audiberti parle de "la noirceur secrète du lait" " 12 , et il montre que cette catalyse est de droit pour le style concessif, lequel a la charge de veiller à ce que l'événement ne vienne jamais à manquer au discours, à la pulsion discursive.

Cet hommage à la divination de Bachelard était indispensable pour poser et démêler le destin de l'obscur dans la peinture européenne. En premier lieu, qu'il s'agisse d'une évolution stylistique collective ou individuelle, l'on constate que la direction est celle, relativement inattendue, de l'obscurcissement, que ce soit dans le passage de l'art de la Renaissance à l'art baroque, ce que Wölfflin désigne comme le déplacement - déjà évoqué - de la "clarté absolue» vers la "clarté relative» 13 , ou bien pour telle évolution personnelle, par exemple de M. Rothko, dont le parcours à partir de 1957 est reconnu comme un assombrissement, puis, si l'on ose dire, comme un noircissement ${ }^{14}$.

Le commerce de la lumière et de l'ombre suppose un émetteur puissant, une continuité spatiale et temporelle, une grandeur meuble, c'est-à-dire ayant vocation à circuler, un transfert attesté par un observateur digne de foi, enfin un récepteur approprié. L'ombre apparait quand l'un de ces ingrédients au moins fait défaut ou s'avère insuffisant; l'ombre est grosso modo le négatif de la lumière. Cette identification est mise en discours par le style implicatif: pas de source lumineuse, pas de lumière et, du point de vue actantiel, l'ombre devient un attracteur-négateur, un «trou noir» où se perd la lumière si elle pointe. Pour le style concessif, les choses se passent tout autrement: loin de combattre la lumière, l'ombre dans le plan du contenu, le noir dans le plan de l'expression deviennent des émetteurs de lumière; contre toutes attentes, c'est-à-dire celles qui sont solidaires du style implicatif, l'ombre émane la lumière, le noir recèle la couleur. Pour appuyer ce renversement, G. Bachelard cite successivement E. Guillevic et M. Leiris: 
Au fond du bleu il y a le jaune,

Et au fond du jaune il y a le noir,

Du noir qui se lève

Et qui regarde,

Qu'on ne pourra pas abattre comme un homme

Avec ses poings.

La couleur noire, dit aussi Michel Leiris (Aurora), «loin d'être celle $d u$ vide et $d u$ néant, est bien plutôt la teinte active qui fait saillir la substance profonde, et, par conséquent, sombre de toutes choses». 15

Le passage d'un état à un autre, ici d'un ton à un autre, entre, très raisonnablement, dans la dépendance d'un changement de tonicité selon notre propre convention terminologique. Le progrès immanent à tout continuum stable, c'est-à-dire la saisie par le sujet sensible du plus et du moins, émane une discontinuité, un avant et un après, un ici et un là, un comme-ci et un comme-ça, et cette "morpho-création" (Michaux) est rabattue sur les procès qui entrent dans le champ de présence du sujet. Ainsi que le souligne Bachelard: «[...] Rouge est plus près de rougir que de rougeur $[. . .]_{»}{ }^{16}$. Et Gothe ne pense pas autrement, puisque, à partir de la polarité [jaune versus bleu], il «déduit» les autres couleurs par «intensification» de leur principe interne:

(i) 772 - Aucune couleur ne pouvant être considérée comme stabilisée, on peut très facilement, en l'épaississant et en l'assombrissant, intensifier le jaune et l'élever en direction $d u$ rouge. La couleur augmente en énergie et apparaît dans l'orange plus puissante et plus magnifique. 17

(ii) Le bleu s'intensifie d'abord en indigo et en mauve, puis en violet, mais Gœthe estime, dans le fragment 790, qu' "[...] un violet saturé tout à fait pur serait en quelque sorte une présence insupportable». (iii) Enfin, en raison de la subordination du caractère au ton dans la terminologie de J.Itten, l'ascendance visée et éprouvée par Gothe reçoit le pourpre, le magenta aujourd'hui, comme l'oméga associant les sur-contraires propres à chaque série:

794 - [...] Et ainsi, lors des phénomènes physiques, ce phénomène coloré le plus élevé de tous apparaît par la fusion de deux termes opposés qui se sont progressivement préparés euxmêmes à se réunir. 18

L'articulation des catégories chromatiques dans le Traité des couleurs est donc justiciable de l'ascendance:

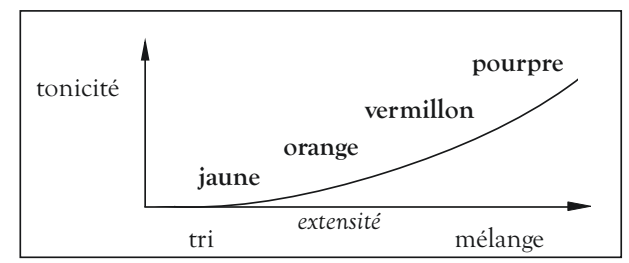

Dans cette perspective, le chromatisme se déploie, pour le plan de l'expression, dans un espace à deux dimensions, mais, pour le plan du contenu, la référence à la temporalité n'est pas moins décisive; et dès lors on ne s'étonne pas de voir Tanizaki lui aussi penser le chromatisme comme une émergence:

[...] Car un laque décoré à la poudre d'or n'est pas fait pour être embrassé d'un seul coup d'œil dans un endroit illuminé, mais pour être deviné dans un lieu obscur, dans une lueur diffuse qui, par instants, en révèle l'un ou l'autre détail [...]. 19

Dans le même ordre d'idées, la technique de Rothko consiste, à partir de superpositions exprimées, à manifester des transparences et des traversées.

Il suffira donc qu'une concentration d'«intensité» pour Michaux ${ }^{20}$, de tonicité pour nous, se laisse reconnaitre pour qu'une poïèse miraculeuse, c'est-àdire concessive, soit aussitôt affirmée en discours. Dans La Peau de chagrin, Balzac, qualifié par Baudelaire de "visionnaire passionné», décrit la photopoièse, l'émergence de la luminosité, à partir de la noirceur du chagrin:

[...] il [Raphaël de Valentin] se pencha pour le regarder alternativement sous toutes les faces; et alors, il découvrit bientôt une cause naturelle à cette lucidité. Les grains noirs $d u$ chagrin étaient si soigneusement polis et si merveilleusement brunis, les rayures capricieuses en étaient si propres et si nettes que, pareilles à des facettes de grenat, les aspérités de ce cuir oriental simulaient autant de petits foyers qui réfléchissaient vivement la lumière. 21 
Il est clair que l'intensification gœthéenne au principe du chromatisme est obtenue dans ce texte par le grossissement résultant du passage de la vue de loin à la vue rapprochée: «il se pencha [...] et alors, il découvrit bientôt $[\ldots] »$.

De façon inattendue pour la doxa accrochée à ses stéréotypes, l'épisode du chagrin dans le roman mythique de Balzac se révèle en concordance tensive avec le fragment d'Éloge de l'ombre analysé par Greimas dans De l'imperfection. Pour Tanizaki, l'obscur contient et dérobe à la vue le lumineux, mais de temps à autre une épiphanie providentielle vient rappeler à l'observateur qu'il est comme en état de cécité:

Avez-vous jamais, vous qui me lisez, vu «la couleur des ténèbres

à la lueur d'une flamme»? Elles sont faites d'une matière autre que celle des ténèbres de la nuit sur une route, et si je puis risquer une comparaison, elles paraissent faites de corpuscules comme d'une cendre ténue, donc chaque parcelle resplendirait de toutes les couleurs de l'arc-en-ciel. [...] $]^{22}$

À l'instar du lait pour Audiberti, selon Greimas:

[L']absence de couleur qu'est le noir cache donc une présence bariolée explosive. Les ténèbres parfaites contiennent virtuellement toutes les couleurs, toute la beauté du monde, elles sont la couleur protopathique: rien d'étonnant à ce que l'énergie condensée dans chacune des parcelles «resplendi[sse] de toutes les couleurs de l'arc-en-ciel» et que l'objet apparaisse comme une révélation de l'intimité de l'être. 23

Greimas commente ainsi ce fragment de Tanizaki: Objet esthétique unique, éphémère, aperçu une seule fois dans la vie: son apparition est due, de plus, à un concours de circonstances et non pas à une disposition particulière du sujet; $[\ldots]^{24}$

Ce commentaire de Greimas appelle plusieurs remarques interrogatives. En premier lieu, il fait écho à la réflexion de Wölfflin, quand ce dernier insiste sur la promotion accordée à l'inattendu par le baroque: "[...] c'est dans l'apparition fortuite qu'on cherche l'expressif „ ${ }^{25}$. En forçant le trait, mais la réflexion de Greimas y invite, nous dirons que le «fortuit» est un objet dans une perspective implicative, mais se dénonce comme sujet dans une perspective concessive. Cette conviction est également celle de P. Soulages:

[...] Nous continuons à appeler cela du noir; en réalité, c'est tout autre chose qui est en action, au point que j'ai pu dire que mon outil n'était pas le noir mais la lumière. Ce noir ainsi employé, n'est pas monochrome, il en est même le contraire. 26

Nous y reviendrons.

\subsection{La densité tensive du discours}

Notre recherche aboutit à une aporie définie par la coexistence de deux affirmations contradictoires: $(i)$ jusqu'à l'administration d'un démenti convaincant, les catégories forment une structure indéfectible, c'est-à-dire qu'elles sont dans une relation d'interdépendance; (ii) le devenir des catégories, s'il a lieu, montre une tendance certaine à l'autonomie, une tendance au chacun pour soi. Ainsi, à propos du traitement de la couleur par Delacroix, Baudelaire note:

Il semble que cette couleur, qu'on me pardonne ces subterfuges

de langage pour exprimer des idées fort délicates, pense par ellemême, indépendamment des objets qu'elle habille. ${ }^{27}$

De même, pour Wölfflin: «La composition, la lumière, la couleur n'ont plus désormais pour fonction première de mettre en évidence la forme; elles mènent leur vie propre " ${ }^{28}$. Le texte de Tanizaki suggère une solution relativement simple: si, à partir d'un couple [A-B], la grandeur $[A]$ accède à l'autonomie, cette dernière est de l'ordre du paraître. Du point de vue phénoménologique, la grandeur [B] est virtualisée, enregistrée comme une absence; du point de vue sémiotique, la grandeur [B] est actualisée, enregistrée comme une présence dans l'absence. Autrement dit, le paradigme des modes d'existence 29 permet de comprendre pourquoi et comment le noir peut pour certains devenir le gardien de la couleur.

Dans le même ordre d'idées, et à partir des remarques convergentes de Wölfflin et de Greimas, nous aimerions introduire le concept de densité tensive. Le discours, pour des motifs variés et inégaux, est souvent en deçà de lui-même et ce souci d'économie 
conduit à un usage non rhétorique de la synecdoque, ce qui signifie que le discours n'exprime pas forcément toutes les sub-valences prévues par l'hypothèse du schématisme tensif. Lorsque Greimas écrit: «Objet esthétique unique, éphémère, aperçu une seule fois dans la vie: son apparition est due, de plus, à un concours de circonstances et non pas à une disposition particulière du sujet", l'accent est porté pour ce fragment sur le couple de sub-valences: [tempo vif $\rightarrow$ temporalité brève]. Toutefois l'appartenance des quatre ordres de sub-valences à une structure autorise une catalyse des sub-valences non manifestées, c'est-àdire le rapatriement des sub-valences reconnues comme défectives, ici de tonicité et de spatialité, à l'intérieur du champ discursif. La densité tensive propre au fragment de Greimas est, sous la convention indiquée, une densité moyenne; la saturation des sub-valences, opérée tant par l'appel à une syllogistique proprement sémiotique que par l'analyse d'autres séquences du texte de Greimas, donne ceci:

\begin{tabular}{|c|c|c|c|c|c|}
\hline \multicolumn{2}{|c|}{$\begin{array}{c}\text { plan de l'expression } \\
\text { tant verbal que } \\
\text { non verbal }\end{array}$} & \multicolumn{2}{|c|}{$\begin{array}{l}\text { émanation de la } \\
\text { lumière par l'ombre } \\
\text { [concession] }\end{array}$} & \multicolumn{2}{|c|}{$\begin{array}{l}\text { absorption de la } \\
\text { lumière par l'ombre } \\
\text { [implication] }\end{array}$} \\
\hline \multirow{4}{*}{$\begin{array}{l}\text { plan du } \\
\text { contenu }\end{array}$} & tempo $\rightarrow$ & vif & \multirow{4}{*}{ 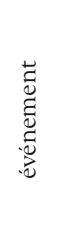 } & lent & \multirow{4}{*}{ 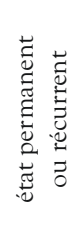 } \\
\hline & tonicité $\rightarrow$ & tonalisant & & atonisant & \\
\hline & temporalité $\rightarrow$ & bref & & long & \\
\hline & spatialité $\rightarrow$ & localisé & & répandu & \\
\hline
\end{tabular}

La poétique concessive de la noirceur transcende la noirceur; la noirceur elle-même ne fournit pas: elle reçoit du fait de sa concentration, de son titre de terme intensif, de la reconnaissance de son exclusivité: «Objet esthétique unique, éphémère, aperçu une seule fois dans la vie» selon Greimas, c'està-dire qu'elle fonctionne dans le discours comme une valeur d'absolu. Sa poétique n'est donc pas celle de la noirceur, mais celle - mythique - des valeurs d'absolu, si bien que la noirceur elle-même doit être rendue au plan de l'expression.

\section{LA TONICITÉ RÉFLECTRICE}

Après avoir exploré la tonicité émettrice, nous envisageons maintenant, ainsi que nous l'avons annoncé, la tonicité réflectrice. Dans ces deux quatrains du poème intitulé Rêve parisien:
Et tout, même la couleur noire
Semblait fourbi, clair, irisé;
Le liquide enchâssait sa gloire
Dans le rayon cristallisé.
Nul astre d'ailleurs, nuls vestiges
De soleil, même au bas du ciel,
Pour illuminer ces prodiges,
Qui brillaient d'un feu personnel!

Baudelaire résume la problématique propre à l'actantialisation de la lumière. De deux choses l'une: ou bien le récepteur s'en tient strictement à sa fonction, ou bien il renvoie la lumière et il devient un récepteur-émetteur; il brille. L'opposition pertinente est donc, une fois de plus, entre le et et le ou: [émetteur vs récepteur] versus [émetteur vs émetteurrécepteur]. Cette distinction est à la merci du syncrétisme que la définition du Micro-Robert inclut dans sa définition: «Briller ou réfléchir une lumière vive». Nous retenons /brillant/ comme un surcontraire tonique de la tonicité réflectrice et, par continuité de convention terminologique, comme un terme $\left[s_{1}\right]$.

\subsection{Une architecture tensive?}

L'embarras du Micro-Robert est également celui de Tanizaki quand il se propose de distinguer l'une de l'autre, à propos du traitement de la lumière par l'architecture traditionnelle japonaise, la montée de l'obscurité, du ressort de la tonicité émettrice, et la montée de la matité, du ressort de la tonicité réflectrice. L'exécution de la première tâche est confiée au toit et surtout à l'auvent :

À l'opposé [des cathédrales gothiques d'Occident], les édifices religieux de notre pays, les bâtiments de notre pays sont écrasés par les énormes tuiles faîtières, et leur structure disparaît tout entière dans l'ombre profonde que projettent les auvents. 30 
Si l'auvent est le sujet opérateur prenant en charge le programme d'atténuation de la lumière externe, le programme d'amenuisement de la lumière interne est confié à deux sujets opérateurs distincts: les shôji ${ }^{31}$ et les murs. À propos des premiers, Tanizaki distingue encore entre ce qui revient au toucher et ce qui revient à la vue:

[...] Et pour que cette lumière épuisée, atténuée, précaire, imprègne à fond les murs de la pièce, ces murs sablés, nous les peignons de couleurs neutres, à dessein; [...] les murs des pièces d'habitation sont presque toujours sablés, et bien rarement luisants. 32

La fonction confiée aux shôji est une opération canonique de tri. Entre l'émetteur et le récepteur vient s'intercaler un dispositif complexe, visant à virtualiser la transparence afin de ne conserver que la translucidité. Nous disons cette opération canonique dans la mesure où elle a été effectuée dans un univers de discours qu'on ne peut même pas dire opposé, tant il est lointain, étranger au Japon de Tanizaki. Selon une métaphore chère à Proust, il appartient à une autre «planète»; il s'agit de l'église romane de Conques, étudiée par M. Renoue, et telle qu'elle s'offre au visiteur après la pose des vitraux voulus par P. Soulages.: «[...] la transparence a été éliminée et la lumière pouvait encore passer ${ }^{33}$. La direction sémiotique n'est pas la même: si la maison japonaise opte pour la décadence de la lumière solaire, dans le cas de l'église la pose des vitraux ne vise pas l'atténuation: «Il [le verre] devait laisser une grande quantité de lumière extérieure, en limiter le moins possible l'intensité» 34 . Dans le cas de la maison japonaise, c'est la perfectivité, la perfection de l'amenuisement, la pénombre, que le Micro-Robert définit comme une "lumière faible, tamisée», qui devient le programme de base.

Le traitement ascendant de la matité connaît lui aussi la scansion aspectuelle fondée sur la distinction entre le relèvement, qui assure la première phase du procès, et le redoublement, qui se charge de la seconde; ces termes font signe à une sémiotique de l'énergie intime et de la fatigue, à une sémiotique du "coup de reins», demeurée contraignante aussi longtemps que l'homme n'a pas disposé de prothèses efficaces. Cette progression est corrélée à une commutation de l'observateur: le sujet sensible à la "surface des murs de couleur crépusculaire» se tient à une certaine distance, en recul à l'égard de l'objet, tandis que le sujet appréciant les «murs presque toujours sablés» a, selon l'expression en usage, le «nez dessus». Pour conserver à cette «lumière indigente» son cachet concessif, il est indispensable que le récepteur luimême n'émette en retour aucune lumière:

\begin{tabular}{|l|c|c|c|}
\hline \multicolumn{3}{|c|}{ décadence $\rightarrow$} \\
\hline \multicolumn{2}{|c|}{ décadence de la lumière } & \multicolumn{2}{|c|}{ ascendance de la matité } \\
\hline atténuation & l'amenuisement & relèvement & redoublement \\
l'auvent & le shôji & $\begin{array}{c}\text { la neutralité } \\
\text { de la couleur }\end{array}$ & $\begin{array}{c}\text { le sablage } \\
\text { des murs }\end{array}$ \\
\hline
\end{tabular}

Ces données sont des «variétés» dans la dépendance de la délicatesse et de la subtilité souhaitées pour l'analyse. La décadence de la lumière dans Éloge de l'ombre ajoute au shôji un second terme qui accomplit l'amenuisement que celui-là a amorcé, c'est le toko no ma:

Nous avons enfin, dans nos pièces de séjour, ce renfoncement qu'on appelle le toko no ma, que nous ornons d'une peinture, d'un arrangement floral, mais la fonction essentielle de cette peinture, n'est pas décorative en soi, car il s'agit plutôt d'ajouter à l'ombre une dimension dans le sens de la profondeur. 35

Du point de vue tensif, l'interprétation la plus simple consiste à confier au shôji l'amenuisement de la lumière et au toko no ma le redoublement de l'obscurité. Dans la mesure où les qualités ne sont, au terme de l'analyse, que des degrés de quantité, un paradigme pertinent n'oppose pas: il gradue, et c'est bien ainsi que Tanizaki l'entend:

Si l'on comparait une pièce d'habitation japonaise à un dessin à l'encre de Chine, les shôji correspondraient à la partie où l'encre est la plus diluée, le toko no ma à l'endroit où elle est la plus épaisse. 36 
Tanizaki a une conscience aiguë de l'ambivalence du sens au plan paradigmatique, du caractère inéluctable de la concession au plan syntaxique, puisque les plus pour l'un sont changés en moins pour l'autre, et inversement: «Or, c'est précisément cette lumière indigente et diffuse qui est le facteur essentiel de la beauté de nos demeures " 37 .

Ainsi, face au Japon ancien, lequel réclame, selon Tanizaki, la croissance de l'ombre et la décroissance de la lumière, l'Occident demande la décroissance de l'ombre et la croissance de la lumière; mais comme ce sont des procès, des devenirs, ce sont la justesse et l'ajustement de leurs tempos respectifs ainsi que l'identification des sujets opérateurs compétents, efficients, qui importent. En effet, sans cette identification des valences et des agents qui les servent, la stabilisation et la pérennité d'un univers de discours deviennent difficilement pensables.

\subsection{La structure élémentaire de la tonicité réflectrice} C'est à partir de ces données que nous proposons d'établir, pour la tonicité réflectrice, la série qui l'informe. Nous recevons /brillant/ et/mat/ comme des sur-contraires, respectivement de tonicité et d'atonie; /mat/ étant pour le Micro-Robert ce «qui n'est pas brillant ou poli», nous sommes fondé à recevoir /terne/ comme le sous-contraire atone, compte tenu de sa définition: ce "qui manque d'éclat, qui reflète peu ou mal la lumière»; nous sommes en présence d'une aspectualité «négative», puisque le /terne/ reflète un peu et le /mat/, pas du tout. Pour le sous-contraire tonique, nous retenons le /satiné/, bien que le Micro-Robert renvoie le /satiné/au toucher, puisqu'il le donne comme "lisse et doux au toucher».

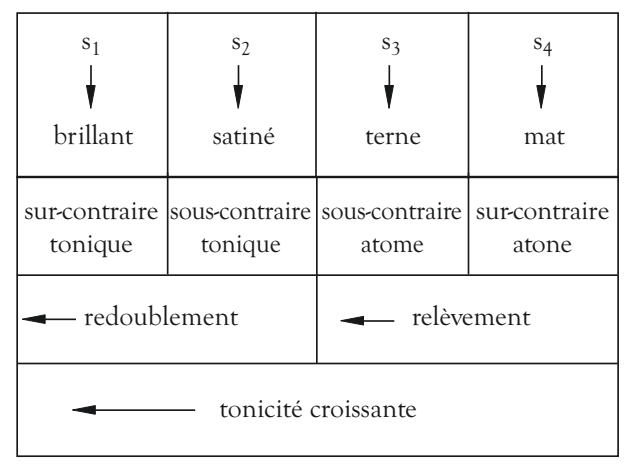

\subsection{Le noir hyperbolique de P. Soulages}

Si l'ouvrage de Tanizaki explore admirablement les arcanes de la matité, l'œuvre peinte de P. Soulages vise la brillance. La tensivité étant pour nous la littéralité même ou, ce qui revient au même, la littéralité étant tensive, il nous suffit de nous mettre à l'écoute de la parole du peintre. Cette préséance que nous accordons au témoignage de l'artiste n'est pas le fait du préjugé, mais une question de syncrétisme. Comme les témoignages concernant Rothko l'affirmaient, ce qui confère à la parole du peintre une autorité - presque toujours - décisive, ce sont la qualité de son attention et la longueur du temps accordée à l'observation de l'effet résultant de ses propres démarches.

Le choix de la couleur noire est pour Soulages le choix de la tonicité "en personne»:

Pour moi, le noir, c'est un excès, une passion... Je n'ai rien éliminé, c'est le contraire: le noir, c'est une couleur violente, elle s'est imposée, elle a dominé, c'est la couleur d'origine. ${ }^{38}$

Et pour notre propos, Soulages suggère une distinction entre le noir selon qu'il est modalisé comme émetteur ou bien comme réflecteur; le peintre le désigne comme l'«outrenoir»:

"Outrenoir» pour dire: au-delà du noir une lumière reflétée, transmutée par le noir. Outrenoir, noir qui cessant de l'être devient émetteur de clarté, de lumière secrète. Outrenoir, un champ mental autre que celui du simple noir [...]. 39

Encore une fois, l'opposition directrice n'opère pas par contraste entre le noir et le blanc, mais par dissimilation du noir, par différenciation tensive entre le "simple noir» et l' "outrenoir», comme s'il importait davantage de s'opposer à soi-même que de s'opposer à l'autre:

\begin{tabular}{|c|c|}
\hline récepteur mat & récepteur brillant \\
\hline «simple noir» & «outrenoir» \\
\hline
\end{tabular}

Selon le peintre lui-même, c'est autant la réflexion optique que la tonicité de la noirceur qui explique son 
choix: «La lumière venant de la toile vers le regardeur crée un espace devant la toile et le regardeur se trouve dans cet espace» ${ }^{40}$. Dans une perspective narrative, le noir tient lieu de programme d'usage et la «lumière qui naît de la toile» de programme de base; dans une perspective sémiosique, le noir s'inscrit dans le plan de l'expression, la lumière qu'il émane dans le plan du contenu:

Nous continuons à appeler cela du noir; en réalité, c'est tout autre chose qui est en action, au point que j'ai pu dire que mon outil n'était pas le noir, mais la lumière. 41

Si la démarche de Soulages est assurément exemplaire par sa rigueur et son invention, son principe était déjà acquis:

Matisse me cite un mot de Pissaro sur Manet, Pissaro lui disant un jour: "Manet est plus fort que nous tous, il a fait de la lumière avec du noir» (ce qui contrecarrait toutes les théories impressionnistes d'alors). 42

La perspective de Soulages est celle de l'efficience et du calcul. Les valences ne sont pas des grandeurs posées, mais des "efficiantes": elles font ou se font ce qu'elles sont dans la mesure où elles sont ce qu'elles font ou se font. Toutes choses étant égales, quand nous retenons pour l'espace la tension [ouvert versus fermé], cette présentation, qui convient à la doxa, n'a pour elle que la commodité et la routine; mais pour rendre sa spécificité sémiotique, il faudrait disposer de termes qui indistinguent entre l'ouvert et l'ouvrant, le fermé et le fermant, à la manière des substantifs mêmes, "ouverture» et «fermeture», lesquels sont, au gré des actes ou des états, ce que sont pour Soulages le temps et l'espace: «L'espace et le temps cessent d'être le milieu dans lequel baignent les formes peintes, ils sont devenus des instruments de la poésie de la toile» ${ }^{43}$. Selon Soulages, en fonction des passages cités, c'est une révolution spatiale affectante qui est visée: le tableau est «bon» si l'informateur d'englobé se change en englobant, si l'espace de l'informateur absorbe, «avale» celui de l'observateur: «la lumière venant de la toile vers le regardeur crée un espace devant la toile et le regardeur se trouve dans cet espace».
Pour l'analyste, l'œuvre de Soulages présente une caractéristique exemplaire, dans la mesure où elle dissocie ce que la peinture traditionnellement compose, à savoir la lumière et la couleur; elle virtualise la couleur et demande à la seule lumière de sensibiliser les valences ${ }^{44}$. Selon la convention terminologique adoptée, la tonicité émettrice opérerait avec les sur-contraires $\left[\mathrm{s}_{1} \& \mathrm{~s}_{4}\right]$, la tonicité réflectrice avec les sous-contraires $\left[s_{2} \& s_{3}\right]$. La présence du point de vue paradigmatique aurait pour assiette un différentiel propre à la dimension ou à la sous-dimension élues. Du point de vue syntagmatique, elle serait subordonnée à un tremblement valenciel, tributaire, le plus souvent mais non exclusivement, des déplacements, des avancées et des reculs de l'observateur, eux-mêmes dirigés par les attentes vécues et les satisfactions éprouvées.

\section{POUR FINIR}

La réflexion calculée de la lumière par le noir devient le plan de l'expression d'une semiosis transculturelle, peut-être transhistorique. Dans La Catégorie des cas, Hjelmslev instruit leur plan du contenu dans les termes suivants: trois dimensions travaillent l'espace dont le sujet s'institue le centre, qui sont respectivement la direction selon [rapprochement versus éloignement], la cohérence, c'est-à-dire le "degré d'intimité avec lequel deux objets sont liés ensemble», ce qui donne lieu à la tension [adhérence versus inhérence], enfin la tension entre la subjectivité et l'objectivité 45 . Le traitement de l'espace plastique ne semble pas faire appel à d'autres catégories. Lorsque Soulages invite l'observateur à se déporter, ou tout au moins à se laisser déporter de son propre espace vers celui de l'informateur, celui du tableau, il «obéit", il reproduit en premier lieu la propension au rapprochement; en second lieu, il substitue l'inhérence à l'adhérence, c'est-à-dire une intériorité "inessive" à une extériorité; enfin, eu égard à la troisième dimension, il accuse le pôle de la subjectivité, puisque le «regardeur» est syncrétiquement, indissociablement, l'agent et le patient. 


\section{NOTES}

1. Du Marsais, Traité des Tropes, Paris, Le Nouveau Commerce, 1977,

p. 14 .

2. P. Fontanier, Les Figures du discours, Paris, Flammarion, 1968, p. 57.

3. L. Hjelmslev, Prolégomènes à une théorie du langage, Paris, Éd. de

Minuit, 1971, p. 49-57. La supériorité de la position hjelmslevienne

tient à ceci que, à la différence du binarisme, elle n'envisage pas les termes, mais les relations alternatives possibles [dépendance vs interdépendance], [réciprocité vs non-réciprocité] entre ces termes. 4. P. Claudel ayant écrit : "Le bleu est l'obscurité devenue visible», Bachelard ose le corriger: "Pour sentir cette image, nous nous permettons de changer le participe passé car dans le règne de l'imagination, il n'y a pas de participe passé. Nous dirons donc: "Le bleu est l'obscurité devenant visible" ". L'Air et les Songes, Paris, J. Corti, 1992, p. 196.

5. J. Itten, Art de la couleur, Paris, Dessain \& Tolra, p. 15.

6. J. Tanizaki, Éloge de l'ombre, Paris, Publications orientalistes de France, 1993, p. 34.

7. Ibid., p. 37. C'est nous qui soulignons.

8. J.W. Gœthe, Traité des couleurs, Paris, Triades, 2000, p. 91. La théorie de Gothe se caractérise par le fait qu'elle place la couleur dans la dépendance de la lumière, qu'elle élit la lumière comme constante et la couleur comme variable: "[...] il faut considérer les couleurs absolument comme des demi-lumières, des demi-ombres" (ibid.). Le gris fonctionne dans ce système comme une preuve péremptoire: «c'est pourquoi, lorsqu'étant mélangées leurs qualités spécifiques se neutralisent réciproquement, elles produisent une teinte d'ombre, un gris" (ibid.).

9. Ibid.

10. J. Tanizaki, op. cit., p. 42.

11. J.W. Gothe, op. cit., p. 104.

12. G. Bachelard, La Terre et les rêveries du repos, Paris, J. Corti, 1992,

p.23. Et Bachelard d'ajouter: "Quel mérite, en effet la neige aurait-elle d'être blanche si sa matière n'est point noire?».

13. Selon H. Wölfflin : “Ce qui est remarquable, c'est que l'époque classique a conçu un idéal de clarté absolue que le XVe siècle n'avait soupçonné que confusément, et que le XVII a délibérément abandonné" (Principes fondamentaux de l'histoire de l'art, Brionne, G. Monfort, 1989, p. 17).

14. Cf. Rothko. Catalogue d'exposition, Paris, Musée d'art moderne, 1999, p. 38 .

15. G. Bachelard, op. cit., p. 27.

16. Ibid., p. 89.

17. J.W. Gœthe, op. cit., p. 268.

18. Ibid., p. 271.

19. I. Tanizaki, op. cit., p. 43.

20. Selon H. Michaux: «Une pensée, une pensée-sentiment est génératrice d'autres pensées, de velléités, d'actions parfois et d'une fugitive transformation générale. Mais, sans une certaine extrême, extrême concentration, il n'y a pas action directe, massive, permanente, magique de cette pensée sur celui qui l'a pensée. Intensité, intensité, intensité dans l'unité, voilà qui est indispensable. Il y a un certain seuil, à partir duquel, mais pas avant, une pensée-sentiment compte, compte autrement, compte vraiment et prend un pouvoir. Elle pourra même rayonner...». Euvres complètes, t. 2, Paris, Gallimard, coll. «Bibliothèque de la Pléiade», 2001, p. 377.
21. H. de Balzac, La Peau de chagrin, Paris, Gallimard, coll. "Le Livre de poche", 1992, p. 54.

22. J. Tanizaki, op. cit. , p. $86-87$.

23. A.G. Greimas, De l'imperfection, Périgueux, P. Fanlac, 1987, p. 51.

24. Ibid., p. 48.

25. H. Wölfflin, op. cit., p. 225.

26. Cité par C. Stoullig, Catalogue de l'exposition Soulages, Paris, Cajarc, 1992.

27. C. Baudelaire, Euvres complètes, Paris, Gallimard, coll.

"Bibliothèque de la Pléiade», 1954, p. 707 (c'est nous qui soulignons).

28. H. Wölfflin, op. cit., p. 17. De même, à propos de la gravure de Rembrandt, Les Pèlerins d'Emmaüs, Wölfflin note: "Que l'on compare cette pièce à une composition comme celle des Pèlerins d'Emmaüs de Dürer, dans sa petite Passion gravée sur bois, et on verra nettement jusqu'à quel point l'éclairage s'est développé ici indépendamment de l'objet pour acquérir une vie propre" (ibid., p. 228).

29. A. J. Greimas et J. Fontanille, Sémiotique des passions, Paris, Éd. du Seuil, 1991, p. 56-60; cf. également J. Fontanille et C. Zilberberg,

Tension et Signification, Liège, P. Mardaga, 1998, p.99-100 et p. 137-139. 30. Ibid., p. 149.

31. Dans le glossaire, le shôji est défini comme une «cloison mobile constituée par une armature de lattes en quadrillage serré, sur laquelle on colle un papier blanc épais qui laisse passer la lumière, mais non le regard. [...] Aujourd'hui les shôji sont le plus souvent précédés, ou même remplacés par des portes vitrées ». (Tanizaki, op. cit., p. 108 - c'est nous qui soulignons.)

32. J. Tanizaki, op. cit. , p. 52.

33. M. Renoue et R. Dengreville, Conques, Rodez, Éd. du Rouergue, 1997 , p. 210.

34. Ibid., p. 208.

35. J. Tanizaki, op. cit., p. 54.

36. Ibid., p. 56

37. Ibid., p. 52.

38. P. Soulages, Catalogue, Toulouse, Les Abattoirs, 2000, p. 102.

39. Ibid.

40. Ibid., p. 103.

41. Cité par C. Stoullig, ibid. (voir note 26).

42. Cité par A. Marchand, dans Matisse, Paris, Éd. Maeght, 1993, p. 24.

43. P. Soulages, op. cit., p. 100.

44. La minutie des analyses de certains tableaux de Soulages par M.

Renoue apporte à notre hypothèse d'ensemble une double confirmation. En premier lieu, toute surface proposée manifeste un différentiel de tempo, éventuellement nul ou mal calculé, même si cette caractérisation doit pour l'instant davantage à l'intuition qu'à l'analyse. Le contraste des à-plats et des stries dans les tableaux de Soulages est pour M. Renoue d'ordre tensif: «Les stries jouent du contraste, de l'opposition entre intensités d'un noir profond, ombreux et d'un noir lumineux pour souligner les formes linéaires données à la matière, quand la surface de l'enduit semble plus sage, moins intensive avec seulement parfois des oppositions moindres et en continu de luminosité" (M. Renoue, " "Des stries et du noir" aux rythmes et aux lumières. Une description de sept peintures de Pierre Soulages ", Protée, vol. 27, no3, hiver 1999-2000, p. 113).

45. L. Hjelmslev, La Catégorie des cas, Munich, W. Fink, 1962, p. 128 129. 